\title{
Mapping Local Optical Densities of States in Finite Si Photonic Structures with Monochromated, Nanoscale Electron Spectroscopy
}

\author{
Judy J. Cha*†, Zongfu Yu**, Eric Smith***, Martin Couillard*†, Shanhui Fan**, David A. Muller* \\ * School of Applied and Engineering Physics, Cornell University, Ithaca, NY 14853 \\ ** Department of Electrical Engineering, Stanford University, Stanford, CA, 94305 \\ *** Department of Physics, Columbia University, New York, NY 10027 \\ $\uparrow$ Current address: Department of Materials Science and Engineering, Stanford University, Stanford, \\ CA, 94305 \\ + Current address: Department of Materials Science and Engineering and Canadian Centre for \\ Electron Microscopy, McMaster University, Hamilton, ON, Canada, L8S 4L7
}

Relativistic electrons travelling in or near a structured dielectric medium generate radiative losses such as Cherenkov and transition radiation that act as a virtual, broadband light source, coupling to the photonic densities of states (DOS) $[1,2,3]$. The effect is most pronounced when the imaginary component of the frequency-dependent dielectric function, $\varepsilon(\omega)$, is zero, a regime where no loss or coupling can occur in a non-retarded, Poisson's formulation of electrodynamics. In contrast to the non-retarded treatment where the loss is derived from and proportional to $\operatorname{Im}[-1 / \varepsilon(\omega)]$, the retarded loss mechanisms are most noticeable when $\operatorname{Im}[\varepsilon(\omega)]<<1$ and the non-retarded contributions are negligible. Even for mildly-relativistic electrons, these effects are large enough to provide a useful, high-spatial resolution probe of photonic nanostructures.

Monochrmoated, spatially-resolved electron energy-loss spectroscopy (EELS) is used to probe local photonic DOS in finite Si photonic structures. With recent improvements in monochromators and system stability, the energy resolution of EELS has improved to reach $100 \mathrm{meV}$, comparable to $\sim 30$ $\mathrm{nm}$ spectral resolution in the visible frequencies. Figure 1 shows the photonic modes of $\sim 1.5 \mu \mathrm{m}-$ thick Si photonic structures, probed with EELS. The experiment agrees well with simulated photonic bands and DOS using the MIT MPB package [4]. Direct, spatial mapping of photonic modes at nanoscale is also performed (Figure 2), showing varying intensity distributions of different photonic modes. Using the focused electron beam, the damping of the photonic modes at the edges of the finite photonic structure is additionally studied, as shown in Figure 3.

Key strengths of EELS for probing photonic structures are its capability to probe photonic modes embedded inside the photonic structures, unlike surface-sensitive scanning near-field optical microscopy, and its superior nanometer-scale resolution. The nanometer resolution of EELS allows a direct mapping of the optical modes of unconventional dielectric systems that may contain defects or extend to only a few hundred nanometers.

\section{References:}

[1] F. J. Garcia de Abajo, et al. Phys. Rev. Lett. 91, 143902 (2003).

[2] A. Yurtsever, M. Couillard, D. A. Muller, Phys. Rev. Lett. 100, 217402 (2008)

[3] F. J. Garcia de Abajo and M. Kociak, Phys. Rev. Lett. 100, 106804 (2008).

[4] S. G. Johnson and J. D. Joannopoulos, Optics Express 8, 173 (2001).

[5] Supported by CCMR, an NSF MRSEC (DMR 0520404) and NSF EEC-0117770, 0646547. 

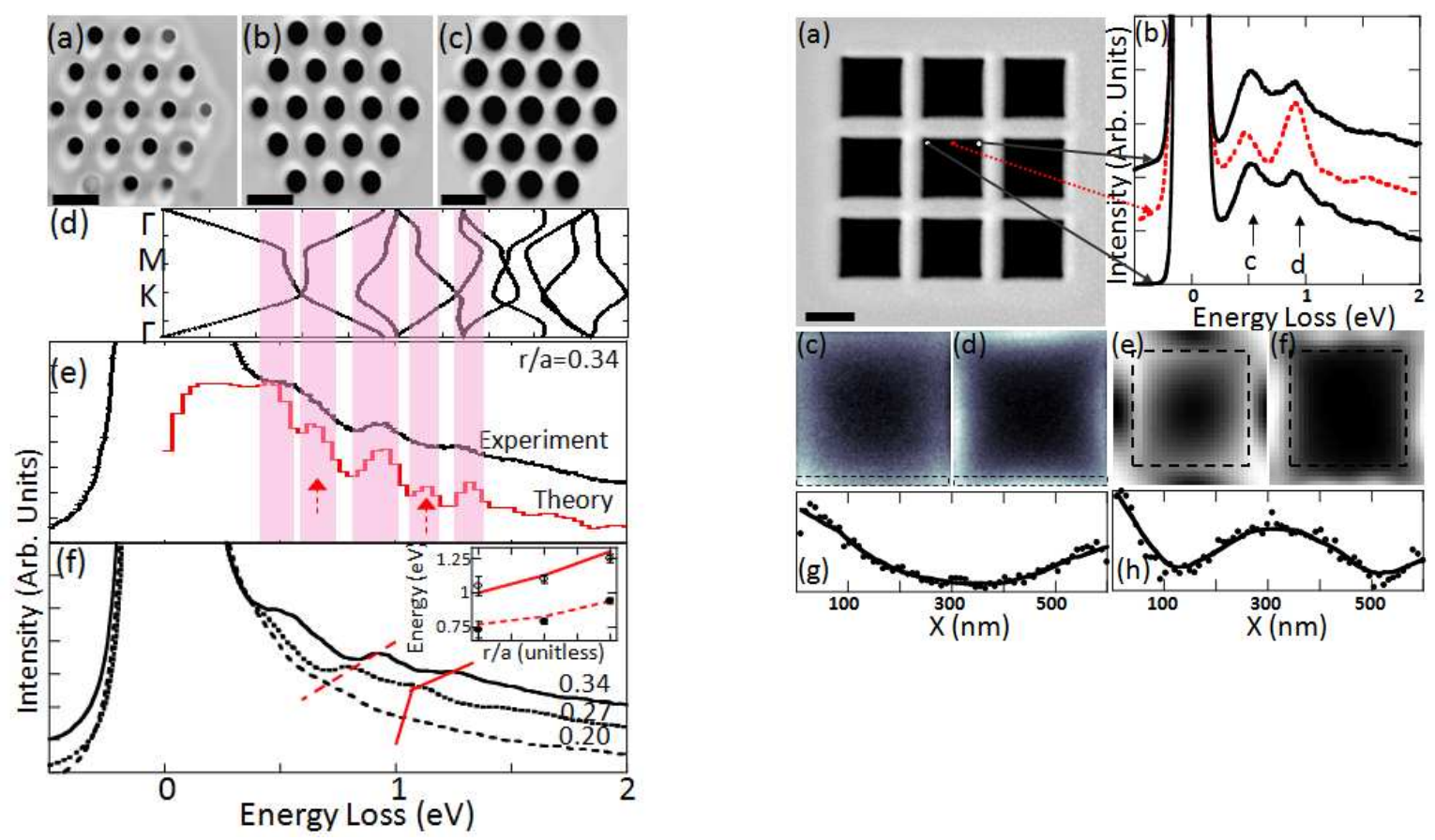

Figure 1 (Left). Si photonic structures of cylindrical holes on a triangular lattice grid with varying r (radius) /a (grid spacing) ratios: (a) 0.20, (b) 0.27, and (c) 0.34. (d) Photonic transverse-magnetic (TM) band calculation of the structure in (c). (e) EELS from the structure in (c) and a simulated photonic DOS. (f) Shifts in the photonic mode energies with varying $r$ a ratios. The inset shows the agreement between the experiment and the simulation. Scale bars $=500 \mathrm{~nm}$.

Figure 2 (Right). Mapping of the photonic modes. (a) shows the photonic structure. Spectra near the top side of the central square are selectively shown in (b). The areas of the two peaks, indicated by the arrows, are integrated to obtain intensity maps of the central square, shown in (c) and (d). (e, f) Calculated field intensity distributions of TM modes, corresponding to the two peaks in (b). (g,h) Averaged line profiles obtained from (c) and (d). Scale bars $=500 \mathrm{~nm}$.

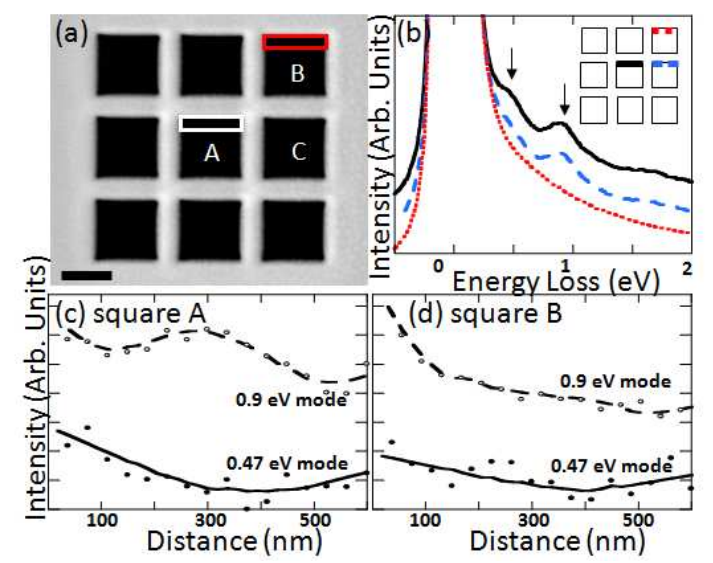

Figure 3. Damped photonic modes near the edge of the finite photonic structure. (a) shows the photonic structure. Scale bar $=500 \mathrm{~nm}$. (b) EELS taken from the top side of squares labeled A, B, and C. (c,d) Intensity profiles of the two peaks, indicated by arrows in (b), obtained from the top side of square $\mathrm{A}$ and $\mathrm{B}$ respectively. 